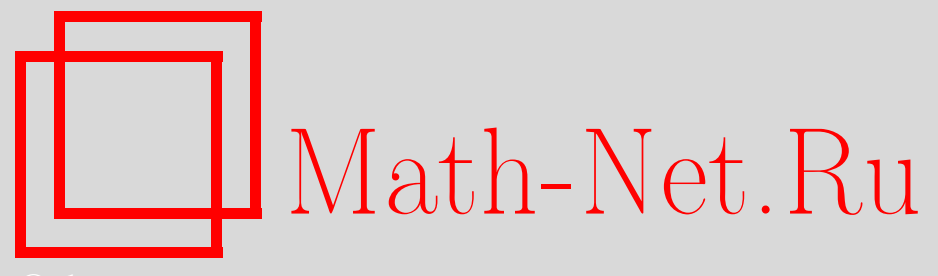

Б. И. Селиванов, Семейство многомерных статистик хиквадрат, Дискрет. матем., 2002, том 14, выпуск 3, 130142

DOI: https://doi.org/10.4213/dm260

Использование Общероссийского математического портала Math-Net.Ru подразумевает, что вы прочитали и согласны с пользовательским соглашением http://www.mathnet.ru/rus/agreement

Параметры загрузки:

IP : 52.87 .193 .239

26 апреля 2023 г., 15:02:04

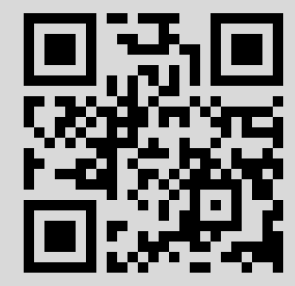




\title{
Семейство многомерных статистик хи-квадрат
}

\author{
() 2002 г. Б. И. Селиванов
}

\begin{abstract}
Рассматривается последовательность независимых испытаний, которая согласно гипотезе $H_{0}$ есть реализация некоторой полиномиальной схемы. Определяется семейство многомерных статистик хи-квадрат, включающее в себя статистики последовательного критерия хи-квадрат. Сформулированы условия, обеспечивающие сходимость распределений рассматриваемых статистик к предельным как при гипотезе $H_{0}$, так и при альтернативах, контигуальных к $H_{0}$. Найден вид преобразований Лапласа предельных распределений статистик введенного семейства.

Работа выполнена при поддержке Российского фонда фундаментальных исследований при Президенте РФ по грантам и государственной поддержке ведущих научных школ, проект 00-15-96136.
\end{abstract}

\section{1. Введение}

Рассмотрим последовательность независимых случайных величин

$$
X_{1}, X_{2}, \ldots, X_{n},
$$

принимающих $N$ значений $1,2, \ldots, N$. Число $N$ в дальнейшем предполагаем фиксированным. Будем предполагать фиксированной также нулевую гипотезу $H_{0}$, согласно которой каждая из случайных величин имеет полиномиальное распределение с вероятностями исходов, равными соответственно $p_{1}, \ldots, p_{N}$, где

$$
0<p_{i}<1, \quad i=1, \ldots, N, \quad \sum_{i=1}^{N} p_{i}=1 .
$$

В работах $[1,2]$ в связи с задачей проверки гипотезы $H_{0}$ введены две различные многомерные статистики хи-квадрат. При условии, что справедлива гипотеза $H_{0}$, в $[1,2]$ с использованием метода, предложенного в [1], для рассматриваемых статистик доказано существование предельных распределений и найдены их преобразования Лапласа. В обоих случаях предельные распределения принадлежат известному классу многомерных центральных распределений хи-квадрат. Для статистики последовательного критерия хиквадрат, определенной в [1], для альтернатив, сближающихся с $H_{0}$, в [1] получено предельное распределение, принадлежащее классу нецентральных многомерных распределений хи-квадрат. Определение многомерных распределений хи-квадрат, как центральных, так и нецентральных, можно найти, например, в [3]. Близкое к нему определение приведено ниже в разделе 3 . 
В разделе 2. настоящей работы определено семейство многомерных статистик хиквадрат, включающее упомянутые статистики из работ [1, 2], и введены альтернативы к $H_{0}$, порождающие, в общем случае, неоднородные последовательности (1) независимых случайных величин. Из множества всех этих альтернатив выделяются альтернативы, контигуальные в смысле Ле Кама (см., например, [4], гл. 6) к гипотезе $H_{0}$. В разделе 2 формулируются также некоторые условия, которые накладываются на параметры статистик семейства хи-квадрат, и доказываются вспомогательные утверждения, используемые в разделе 3 при доказательстве предельных теорем.

В разделе 3 найдены также преобразования Лапласа для предельных распределений статистик рассматриваемого семейства. Показано, что полученные предельные распределения принадлежат классу многомерных распределений хи-квадрат, центральных, если справедлива гипотеза $H_{0}$, и нецентральных, если выполняются альтернативы, контигуальные к $H_{0}$. При этом используется подход, описанный в параграфах 2, 3 работы [1] и применяются некоторые известные результаты о распределениях квадратичных форм от нормальных случайных величин.

\section{2. Определения и вспомогательные результаты}

Зададим $r \geqslant 1$ конечных непустых множеств натуральных чисел $A_{1}, \ldots, A_{r}$. Положим

$$
\begin{gathered}
A=A_{1} \cup \ldots \cup A_{r}, \quad A_{k l}=A_{k} \cap A_{l}, \\
\Delta=|A|, \quad \Delta_{k}=\left|A_{k}\right|, \quad \Delta_{k l}=\left|A_{k l}\right|, \quad k, l=1, \ldots, r,
\end{gathered}
$$

где $|M|$ - число элементов множества $M$. Пусть далее $v_{k i}$ - число появлений исхода $i \in\{1, \ldots, N\}$ в испытаниях (1) с номерами $m \in A_{k}, k=1, \ldots, r$. Введем случайные величины

$$
\tilde{\xi}_{k i}=\frac{1}{\sqrt{\Delta_{k} p_{i}}}\left(v_{k i}-\Delta_{k} p_{i}\right), \quad k=1, \ldots, r, \quad i=1, \ldots, N,
$$

и случайный вектор

$$
\tilde{\xi}=\left(\tilde{\xi}_{11}, \ldots, \tilde{\xi}_{1 N} ; \ldots ; \tilde{\xi}_{r 1}, \ldots, \tilde{\xi}_{r N}\right)
$$

составленный из $r$ блоков

$$
\left(\tilde{\xi}_{k 1}, \ldots, \tilde{\xi}_{k N}\right), \quad k=1, \ldots, r
$$

С использованием случайных величин (2) определим $r$-мерную статистику хи-квадрат

$$
\chi^{2}=\left(\chi_{1}^{2}, \ldots, \chi_{r}^{2}\right)
$$

с компонентами

$$
\chi_{k}^{2}=\sum_{i=1}^{N} \tilde{\xi}_{k i}^{2}=\sum_{i=1}^{N} \frac{1}{\Delta_{k} p_{i}}\left(\nu_{k i}-\Delta_{k} p_{i}\right)^{2}, \quad k=1, \ldots, r
$$

Компоненты статистики $\chi^{2}$ зависят от выбора множеств $A_{1}, \ldots, A_{r}$. Задавая различные наборы этих множеств, получим семейство $r$-мерных статистик хи-квадрат. В дальнейшем, говоря, что задана $r$-мерная статистика $\chi^{2}$, будем иметь ввиду, что заданы и множества $A_{1}, \ldots, A_{r}$, определяющие компоненты $\chi^{2}$. 
При $r \geqslant 2$ статистика (4) позволяет построить, как и в [1], последовательный $r$-кратный критерий хи-квадрат. Частными случаями векторной статистики (4) являются

- классическая статистика $\chi^{2}$ К. Пирсона (при $\left.r=1, A_{1}=\{1, \ldots, n\}\right)$;

- статистика, введенная в [1], в этом случае

$$
A_{k}=\left\{1, \ldots, n_{k}\right\}, \quad k=1, \ldots, r, \quad i \leqslant n_{1}<n_{2}<\ldots<n_{r}, \quad r \geqslant 2 ;
$$

- статистика, введенная в [2] и названная скользящим хи-квадрат, где

$$
A_{k}=\left\{n\left(t_{k}-1\right)+1, \ldots, n\left(t_{k}+s-1\right)\right\}, \quad k=1, \ldots, r,
$$

$r \geqslant 2,1 \leqslant t_{1}<\ldots<t_{r}, n \geqslant 1$ и $s \geqslant 2-$ фиксированные целые числа.

Если множества $A_{1}, \ldots, A_{r}$ попарно не пересекаются, то компоненты статистики (4) независимы.

Рассмотрим последовательность полиномиальных схем с векторами вероятностей

$$
\bar{p}=\left\{\bar{p}_{1}, \bar{p}_{2}, \ldots, \bar{p}_{n}, \ldots\right\}
$$

где для $n=1,2, \ldots$

$$
\bar{p}_{n}=\left(p_{1}(n), \ldots, p_{N}(n)\right), \quad 0 \leqslant p_{i}(n) \leqslant 1, \quad i=1, \ldots, N, \quad \sum_{i=1}^{N} p_{i}(n)=1 .
$$

Будем говорить, что выполняется (простая) гипотеза $H(\bar{p})$, если в $n$-м испытании (1) реализуется полиномиальная схема (7), $n=1,2, \ldots$

В случае

$$
\bar{p}_{1}=\bar{p}_{2}=\ldots=\bar{p}_{n}=\ldots=p, \quad p=\left(p_{1}, \ldots, p_{N}\right)
$$

выполняется гипотеза $H(\bar{p})=H_{0}$. Если же в (6) $\bar{p}_{n} \neq p$ для некоторого $n \geqslant 1$, то гипотезу $H(\bar{p}) \neq H_{0}$ будем называть альтернативой к нулевой гипотезе. В настоящей работе в качестве альтернатив $H(\bar{p})$ мы будем рассматривать лишь те, которые контигуальны в смысле Ле Кама [4] к гипотезе $H_{0}$.

Имеет место следующее предложение, частными случаями которого являются равенства (2), (4) из [1] и лемма 1 из [2].

Лемма 1. Пусть последовательность (1) получена при гипотезе $H(\bar{p})$. Тогда для случайных величин (2) выполняются равенства

$$
\begin{aligned}
\mathbf{E} \tilde{\xi}_{k i} & =\frac{1}{\sqrt{\Delta_{k} p_{i}}} \sum_{m \in A_{k}}\left(p_{i}(m)-p_{i}\right) \\
\operatorname{cov}\left(\tilde{\xi}_{k i}, \tilde{\xi}_{l j}\right) & =\frac{1}{\sqrt{\Delta_{k} \Delta_{l} p_{i} p_{j}}} \sum_{m \in A_{k l}} p_{i}(m)\left(\delta_{i j}-p_{j}(m)\right)
\end{aligned}
$$

где $\delta_{i j}$ - символ Кронекера, $k, l=1, \ldots, r, i, j=1, \ldots, N$.

В частности,

$$
\mathbf{D} \tilde{\xi}_{k i}=\frac{1}{\Delta_{k} p_{i}} \sum_{m \in A_{k}} p_{i}(m)\left(1-p_{i}(m)\right)
$$


Доказательство. Равенства (8) следуют непосредственно из (2). Далее заметим, что

$$
\operatorname{cov}\left(\tilde{\xi}_{k i}, \tilde{\xi}_{l j}\right)=\frac{1}{\sqrt{\Delta_{k} p_{i} \Delta_{l} p_{j}}} \mathbf{E}\left(v_{k i}-\mathbf{E} v_{k i}\right)\left(v_{l j}-\mathbf{E} v_{l j}\right)
$$

и

$$
\mathbf{E}\left(v_{k i}-\mathbf{E} v_{k i}\right)\left(v_{l j}-\mathbf{E} v_{l j}\right)=\sum_{m \in A_{k}, n \in A_{l}} \mathbf{E}\left(I\left\{X_{m}=i\right\}-p_{i}(m)\right)\left(I\left\{X_{n}=j\right\}-p_{j}(n)\right),
$$

где $I\{B\}-$ индикатор события $B$. Если $m \neq n$, то выражение под знаком суммы в правой части (11) равно нулю, и (11) приводится к виду

$$
\mathbf{E}\left(v_{k i}-\mathbf{E} v_{k i}\right)\left(v_{l j}-\mathbf{E} v_{l j}\right)=\sum_{m \in A_{k l}} \mathbf{E}\left(I\left\{X_{m}=i\right\}-p_{i}(m)\right)\left(I\left\{X_{m}=j\right\}-p_{j}(m)\right) .
$$

Отсюда и из (10) следует (9). Лемма доказана.

Следствие 1. Если выполняется гипотеза $H_{0}$, то для случайных величин (2) имеют место равенства

$$
\begin{aligned}
\mathbf{E} \tilde{\xi}_{k i} & =0 \\
\operatorname{cov}\left(\tilde{\xi}_{k i}, \tilde{\xi}_{l j}\right) & =\frac{\Delta_{k l}}{\sqrt{\Delta_{k} \Delta_{l}}}\left(\delta_{i j}-\sqrt{p_{i} p_{j}}\right)
\end{aligned}
$$

где $i, j=1, \ldots, N, k, l=1, \ldots, r$.

Будем предполагать, что имеет место следующая схема серий: каждое из множеств $A_{k}$ зависит от переменной $\omega$, пробегающей значения $1,2, \ldots$ Следовательно, от $\omega$ зависят также величины $\Delta, \Delta_{k}, \Delta_{k l}$, множество $A$ и статистика (4). Кроме того, будем предполагать, что от $\omega$ зависят вероятности $p_{i}(m), i=1, \ldots, N, m=1,2, \ldots$, (см. (7)) и тем самым альтернативы $H(\bar{p})$ к нулевой гипотезе. Эту зависимость от $\omega$ мы не всегда будем явно указывать. Нас интересуют асимптотические при $\omega \rightarrow \infty$ распределения статистик (4) как при гипотезе $H_{0}$, так и при альтернативах $H(\bar{p})$ к гипотезе $H_{0}$.

Пусть выполняются следующие условия $F_{1}, F_{2}$ и $F_{3}$.

(F) Если $\omega \rightarrow \infty$, то $\Delta_{k} \rightarrow \infty, k=1, \ldots, r$.

$\left(F_{2}\right)$ Существуют пределы

$$
\lim _{\omega \rightarrow \infty} \frac{\Delta_{k l}}{\sqrt{\Delta_{k} \Delta_{l}}}=\rho_{k l}, \quad 0 \leqslant \rho_{k l} \leqslant 1, \quad k, l=1, \ldots, r, \quad k \neq l
$$

Заметим, что если $k=l$, то $\rho_{k k}=1, k=1, \ldots, r$, и условие $F_{2}$ заведомо выполняется.

$\left(F_{3}\right)$ Выполняется соотношение

$$
\underset{\omega \rightarrow \infty}{\limsup } \sum_{m=1}^{\Delta} \sum_{i=1}^{N} \frac{1}{p_{i}}\left(p_{i}(m)-p_{i}\right)^{2}<\infty
$$


Смысл условия $F_{3}$ заключается в том, что в силу конечности числа исходов $N$ в испытаниях (1) альтернатива $H(\bar{p})$ будет контигуальна к $H_{0}$ тогда и только тогда, когда выполңяется это условие (см., например, [5], §7). Величина

$$
\Delta=\left|\bigcup_{k=1}^{r} A_{k}\right|
$$

фактически является объемом выборки, с использованием которой строится статистика (4).

Лемма 2. Пусть случайные величины (1) получены в предположении, что верна гипотеза $H_{0}$, и выполняются условия $F_{1}$ и $F_{2}$. Тогда при $\omega \rightarrow \infty$ случайный вектор с компонентами (2) сходится по распределению к $r N$-мерному нормальному случайному вектору

$$
\xi=\left(\xi_{11}, \ldots, \xi_{1 N} ; \ldots ; \xi_{r 1}, \ldots, \xi_{r N}\right)
$$

имеющему нулевой вектор средних значений и ковариачионную матричу $D=\left\|d_{k, i, l, j}\right\|$ порядка $r N$ с элементами

$$
d_{k, i, l, j}=\operatorname{cov}\left(\xi_{k i}, \xi_{l j}\right)=\rho_{k l}\left(\delta_{i j}-\sqrt{p_{i} p_{j}}\right), \quad k, l=1, \ldots, r, \quad i, j=1, \ldots, N .
$$

Доказательство. Для каждого $k=1, \ldots, r$ зададим $N$ векторов вида

$$
\begin{aligned}
z_{k 1} & =\left(\frac{1-p_{1}}{\sqrt{\Delta_{k} p_{1}}},-\frac{p_{2}}{\sqrt{\Delta_{k} p_{2}}}, \ldots,-\frac{p_{N}}{\sqrt{\Delta_{k} p_{N}}}\right), \\
z_{k 2} & =\left(-\frac{p_{1}}{\sqrt{\Delta_{k} p_{1}}}, \frac{1-p_{2}}{\sqrt{\Delta_{k} p_{2}}}, \ldots,-\frac{p_{N}}{\sqrt{\Delta_{k} p_{N}}}\right), \\
& \ldots \\
z_{k N} & =\left(-\frac{p_{1}}{\sqrt{\Delta_{k} p_{1}}},-\frac{p_{2}}{\sqrt{\Delta_{k} p_{2}}}, \ldots, \frac{1-p_{N}}{\sqrt{\Delta_{k} p_{N}}}\right) .
\end{aligned}
$$

Далее, для каждого $n=1,2, \ldots$ определим множество

$$
M(n) \subseteq J_{r}=\{1, \ldots, r\}
$$

включив в $M(n)$ элемент $k \in J_{r}$ тогда и только тогда, когда $n \in A_{k}$. Ясно, что $M(n) \neq \varnothing$ тогда и только тогда, когда $n \in A$, и имеет место равенство

$$
\tilde{\xi}=\sum_{n \in A} \zeta_{n}
$$

(напомним, что $\tilde{\xi}$ - случайный вектор (3)). Каждый вектор $\zeta_{n}$ из правой части (16), как и $\tilde{\xi}$, имеет $r$ блоков по $N$ компонент. Ненулевыми в $\zeta_{n}$ являются лишь $t_{n}=|M(n)|$ блоков с номерами $k \in M(n)$. Если в (1) $X_{n}=i$, то в $\zeta_{n}$ блок с номером $k \in M(n)$ полагаем равным $z_{k i}$-му из векторов (15). Остальные блоки $\zeta_{n}$ нулевые. Таким образом, векторы $\zeta_{n}$ в сумме (16) с вероятностями $p_{1}, \ldots, p_{N}$ принимают $N$ значений, зависящих от множеств $M(n)$.

В силу условия $F_{1}$ число слагаемых в сумме (16) неограниченно возрастает и к вектору $\tilde{\xi}$ применима многомерная центральная предельная теорема (см., например, [6]). Используя лемму 1 и условие $F_{2}$, убеждаемся в справедливости леммы 2. 
Лемма 3. Пусть случайные величины (1) получены в предположении, что верна некоторая альтернатива (6) к $H_{0}$ и справедливы условия $F_{1}, F_{2}$, и $F_{3}$. Тогда при $\omega \rightarrow \infty$ случайный вектор

$$
\xi=\left(\xi_{11}^{(1)}, \ldots, \xi_{1 N}^{(1)} ; \ldots ; \xi_{r 1}^{(1)}, \ldots, \xi_{r N}^{(1)}\right)
$$

с компонентами

$$
\xi_{k i}^{(1)}=\frac{v_{k i}-\sum_{m \in A_{k}} p_{i}(m)}{\sqrt{\Delta_{k} p_{i}}}, \quad k=1, \ldots, r, \quad i=1, \ldots, N,
$$

сходится по распределению к $r N$-мерному нормальному случайному вектору (13), определенному в формулировке леммы 2.

Доказательство. Покажем, что для случайных величин (18) выполняются соотношения

$$
\lim _{\omega \rightarrow \infty} \operatorname{cov}\left(\xi_{k i}^{(1)}, \xi_{l j}^{(1)}\right)=d_{k, i, l, j}, \quad k, l=1, \ldots, r, \quad i, j=1, \ldots, N
$$

где величины $d_{k, i, l, j}$ определяются равенствами (14). Зафиксировав $k, l, i$ и $j, 1 \leqslant k, l \leqslant r$, $1 \leqslant i, j \leqslant N$, напишем неравенство

$$
\begin{aligned}
\left|\operatorname{cov}\left(\xi_{k i}^{(1)}, \xi_{l j}^{(1)}\right)-d_{k, i, l, j}\right| \leqslant\left|\operatorname{cov}\left(\xi_{k i}^{(1)}, \xi_{l j}^{(1)}\right)-\frac{\Delta_{k l}}{\sqrt{\Delta_{k} \Delta_{l}}}\left(\delta_{i j}-\sqrt{p_{i} p_{j}}\right)\right| \\
+\left|\frac{\Delta_{k l}}{\sqrt{\Delta_{k} \Delta_{l}}}-\rho_{k l}\right|\left|\delta_{i j}-\sqrt{p_{i} p_{j}}\right| .
\end{aligned}
$$

В силу условия $F_{2}$ при $\omega \rightarrow \infty$

$$
\left|\frac{\Delta_{k l}}{\sqrt{\Delta_{k} \Delta_{l}}}-\rho_{k l}\right|\left|\delta_{i j}-\sqrt{p_{i} p_{j}}\right| \rightarrow 0 .
$$

Далее, учитывая, что $\operatorname{cov}\left(\xi_{k i}^{(1)}, \xi_{l j}^{(1)}\right)=\operatorname{cov}\left(\tilde{\xi}_{k i}, \tilde{\xi}_{l j}\right)$, где случайные величины $\xi_{k i}^{(1)}, \xi_{l j}^{(1)}$ и $\tilde{\xi}_{k i}, \tilde{\xi}_{l j}$ определяются равенствами (18) и (2), соответственно, и используя лемму 1 , получим для первого слагаемого из правой части неравенства (20) оценку

$$
\left|\operatorname{cov}\left(\xi_{k i}^{(1)}, \xi_{l j}^{(1)}\right)-\frac{\Delta_{k l}}{\Delta_{k} \Delta_{l}}\left(\delta_{i j}-\sqrt{p_{i} p_{j}}\right)\right| \leqslant \Sigma_{1}+\Sigma_{2}+\Sigma_{3},
$$

где

$$
\begin{aligned}
& \Sigma_{1}=\frac{1}{\sqrt{\Delta_{k} \Delta_{l} p_{i} p_{j}}} \sum_{m \in A_{k l}} p_{i}(m)\left|p_{j}(m)-p_{j}\right|, \\
& \Sigma_{2}=\frac{1}{\sqrt{\Delta_{k} \Delta_{l} p_{i} p_{j}}} \sum_{m \in A_{k l}} p_{j}(m)\left|p_{i}(m)-p_{i}\right|, \\
& \Sigma_{3}=\frac{1}{\sqrt{\Delta_{k} \Delta_{l} p_{i} p_{j}}} \sum_{m \in A_{k l}} \delta_{i j}\left|p_{i}(m)-p_{i}\right| .
\end{aligned}
$$


Докажем, что правая часть неравенства (22) при $\omega \rightarrow \infty$ стремится к нулю. Рассмотрим, например, слагаемое $\Sigma_{1}$. Применяя неравенство Коши-Буняковского и учитывая, что $\Delta_{k l} / \Delta_{k} \leqslant 1$, получаем оценку

$$
\Sigma_{1} \leqslant\left(\frac{K_{0}}{\Delta_{l}} \sum_{m \in A_{k l}} \frac{1}{p_{j}}\left(p_{j}(m)-p_{j}\right)^{2}\right)^{1 / 2},
$$

где

$$
K_{0}=\max _{1 \leqslant i \leqslant N}\left(1 / p_{i}\right), \quad 0<K_{0}<\infty .
$$

Воспользуемся теперь условием $F_{3}$, из которого следует существование константы $K>0$ такой, что для всех $j=1, \ldots, N$ выполняются неравенства

$$
\sum_{m \in A_{k l}} \frac{1}{p_{j}}\left(p_{j}(m)-p_{j}\right)^{2}<K .
$$

Отсюда в силу $F_{1}$ и оценки (23) вытекает, что при $\omega \rightarrow \infty$

$$
\Sigma_{1} \rightarrow 0 \text {. }
$$

Аналогично доказываем, что при $\omega \rightarrow \infty$

$$
\Sigma_{2} \rightarrow 0, \quad \Sigma_{3} \rightarrow 0
$$

В итоге из (20), (21), (22), (24) и (25) следует справедливость соотношения (19).

Оставшаяся часть доказательства леммы 3 , касающаяся асимптотической нормальности вектора (17), проводится так же, как и в лемме 2.

\section{3. Предельные теоремы}

Приведем определение многомерного распределения хи-квадрат, аналогичное определению из [3].

Обозначим через $N_{r}(a, R) r$-мерное нормальное распределение с вектором средних значений $a$ и ковариационной матрицей $R$. Пусть даны $s \geqslant 1$ независимых случайных векторов $u_{i}=\left(u_{i 1}, \ldots, u_{i r}\right), i=1, \ldots, s$, и $u_{i}$ имеет распределение $N_{r}\left(a_{i}, R\right)$, где матрища $R$ неотрицательно определена и $a_{i}=\left(a_{i 1}, \ldots, a_{i r}\right), i=1, \ldots, r$. Тогда, если $a_{1}=\ldots=$ $a_{s}=0$, то распределение вектора

$$
v=\left(v_{1}, \ldots, v_{r}\right), \quad v_{k}=\sum_{i=1}^{s} u_{i k}^{2}, \quad k=1, \ldots, r,
$$

называется $r$-мерным центральным распределением хи-квадрат. Если же не все векторы $a_{1}, \ldots, a_{s}$ являются нулевыми, то распределение $v$ называется нецентральным распределением хи-квадрат.

Отличие данного определения от того, которое можно найти в [3], заключается в отсутствии требования положительной определенности ковариационной матрищы случайного вектора $u_{1}, \ldots, u_{s}$.

Обозначим $I$ единичную матрицу порядка $r$ и пусть $T=\operatorname{diag}\left\{t_{1}, \ldots, t_{r}\right\}$, где $t_{k} \geqslant 0$, $k=1, \ldots, r$. Определитель матрицы $B$ будем обозначать $|B|$, а штрих у вектора будет означать его транспонирование. 
Лемма 4. Для преобразования Лапласа

$$
l\left(t_{1}, \ldots, t_{r}\right)=\mathbf{E} \exp \left\{-\sum_{k=1}^{r} t_{k} v_{k}\right\}
$$

случайного вектора (26) справедливы представления

$$
\begin{aligned}
l\left(t_{1}, \ldots, t_{r}\right) & =|I+2 T R|^{-s / 2} \exp \left\{-\sum_{i=1}^{s} a_{i} Q a_{i}^{\prime}\right\} \\
& =|I+2 T R|^{-s / 2} \exp \left\{-\sum_{k, l=1}^{r} \alpha_{k l} q_{k l}\right\},
\end{aligned}
$$

где

$$
\begin{gathered}
\alpha_{k l}=\sum_{i=1}^{r} a_{i k} a_{i l}, \quad k, l=1, \ldots, r, \\
Q=\left\|q_{k l}\right\|_{k, l=1}^{r}=(I+2 T R)^{-1} T .
\end{gathered}
$$

Доказательство. В силу независимости случайных векторов $u_{1}, \ldots, u_{s}$ из (26) и (27) вытекает, что

$$
l\left(t_{1}, \ldots, t_{r}\right)=\prod_{i=1}^{s} \mathbf{E} e^{-u_{i} T u_{i}^{\prime}}
$$

Применим к сомножителям из правой части (32) теорему из [7] (см. [7], с. 103) и получим равенство (28), где $Q_{r}$ - матрица (31). Так как

$$
a_{i} Q a_{i}^{\prime}=\sum_{k, l=1}^{r} a_{i k} a_{i l} q_{k l}, \quad i=1, \ldots, s,
$$

из (28) следует (29).

Формулу (28) в случае положительной определенности матрицы $R$ можно найти в [3].

Согласно лемме 4 многомерное центральное распределение хи-квадрат задается параметрами $r, s$ и матрицей $R$, а многомерное нецентральное хи-квадрат распределение, кроме того, зависит от $r(r+1) / 2$ величин $\alpha_{k l}$, удовлетворяющих соотношениям (30). Эти соотношения, если их рассматривать как уравнения относительно неизвестных $a_{i k}$, $i=1, \ldots, s, k=1, \ldots, r$, могут быть несовместны или иметь несколько решений, каждому из которых соответствует набор нормальных случайных векторов $u_{1}, \ldots, u_{s} \mathrm{c}$ ковариационной матрицей $R$.

Теорема 1. Пусть случайные величины (1) получены в предположении, что верна гипотеза $H_{0}$, и выполняются условия $F_{1} u F_{2}$. Тогда при $\omega \rightarrow \infty$ статистика (4) имеет предельное распределение, преобразование Лапласа которого имеет вид

$$
L_{r}\left(t_{1}, \ldots, t_{r}\right)=|I+2 T R|^{-(N-1) / 2},
$$

где $t_{1}, \ldots, t_{r} \geqslant 0, R=\left\|\rho_{k l}\right\|_{k, l=1}^{r}$ и числа $\rho_{k l}$ определены условием $F_{2}$. 
Доказательство. Используя лемму 2 и векторную версию предложения XII из [8] (см. [8], п. 2, с. 4), заключаем, что при $\omega \rightarrow \infty$ статистика $\chi^{2}$ имеет некоторое предельное распределение, для преобразования Лапласа которого имеет место равенство

$$
\begin{aligned}
L_{r}\left(t_{1}, \ldots, t_{r}\right) & =\lim _{\omega \rightarrow \infty} \mathbf{E} \exp \left\{-\sum_{k=1}^{r} t_{k} \chi_{k}^{2}\right\} \\
& =\mathbf{E} \exp \left\{-\sum_{k=1}^{r} t_{k} \sum_{i=1}^{N} \xi_{k i}^{2}\right\},
\end{aligned}
$$

где $\xi_{k i}$ - компоненты вектора (13). Действуя, как в [1], зададим ортогональную матрицу $C=\left\|c_{i j}\right\|_{i, j=1}^{N}$ порядка $N$, элементы последней строки которой определяются равенствами $c_{N j}=\sqrt{p_{j}}, j=1, \ldots, N$, и рассмотрим случайные векторы

$$
\eta_{i}=\left(\eta_{i 1}, \ldots, \eta_{i r}\right), \quad i=1, \ldots, N
$$

где

$$
\eta_{i k}=\sum_{j=1}^{N} c_{i j} \xi_{k j}, \quad k=1, \ldots, r
$$

В условиях теоремы 6 выполняется гипотеза $H_{0}$ и верна лемма 2 . В силу этой леммы вектор $\eta_{N}$ с вероятностью 1 равен нулю, а векторы $\eta_{1}, \ldots, \eta_{N-1}$ независимы, и каждый из них имеет распределение $N_{r}(0, R)$, так что матрица $R$ неотрицательно определена. Это вытекает из того, что аналогично формуле (14) из [2] справедливы равенства

$$
\operatorname{cov}\left(\eta_{i k}, \eta_{j l}\right)=\rho_{k l}\left(\delta_{i j}-\delta_{i N} \delta_{j N}\right), \quad k, l=1, \ldots, r, \quad i, j=1, \ldots, N .
$$

Из (34) и ортогональности матрицы $C$ находим, что

$$
L_{r}\left(t_{1}, \ldots, t_{r}\right)=\mathbf{E} \exp \left\{-\sum_{k=1}^{r} t_{k} \sum_{i=1}^{N-1} \eta_{i k}^{2}\right\} .
$$

Применяя к векторам $\eta_{1}, \ldots, \eta_{N-1}$ лемму 4, из (36) получаем представление (33). Теорема доказана.

Из (33) и леммы 4 следует, что предельное распределение статистики $\chi^{2}$, полученное в теореме 1, есть $r$-мерное центральное распределение хи-квадрат с параметрами $r, N-1$ и матрищей $R=\left\|\rho_{k l}\right\|_{k, l=1}^{r}$, элементы которой определяются условием $F_{2}$.

Теорема 2. Пусть для каждого $\omega=1,2 \ldots$ случайные величины (1) получены в предположении, что справедлива альтернатива $H(\bar{p})=H\left(\bar{p}_{\omega}\right)$ и выполнены условия $F_{1}, F_{2} u$ $F_{3}$. Пусть существуют предель

$$
\lim _{\omega \rightarrow \infty} \sum_{i=1}^{N} \lambda_{k i}(\omega) \lambda_{l j}(\omega)=\alpha_{k l}, \quad k, l=1, \ldots, r, \quad k \leqslant l
$$

где

$$
\lambda_{k i}(\omega)=\frac{1}{\sqrt{\Delta_{k} p_{i}}} \sum_{m \in A_{k}}\left(p_{i}(m)-p_{i}\right), \quad k=1, \ldots, r, \quad i=1, \ldots, N
$$


Тогда при $\omega \rightarrow \infty$ статистика (4) сходится по распределению и преобразование Лапласа ее предельного распределения есть

$$
L_{r}\left(t_{1}, \ldots, t_{r}\right)=|I+2 T R|^{-(N-1) / 2} \exp \left\{-\sum_{k, l=1}^{r} \alpha_{k l} q_{k l}\right\},
$$

где $t_{k} \geqslant 0, k=1, \ldots, r, q_{k l}, k, l=1, \ldots, r,-$ элементы матричы (31), причем $R=$ $\left\|\rho_{k l}\right\|_{k, l=1}^{r} u \alpha_{l k}=\alpha_{k l}, k, l=1, \ldots, r, k \leqslant l$.

Доказательство. Запишем компоненты случайного вектора (3) в виде

$$
\tilde{\xi}_{k i}=\xi_{k i}^{(1)}+\lambda_{k i}(\omega), \quad k=1, \ldots, r, \quad i=1, \ldots, N
$$

(величины $\xi_{k i}^{(1)}$ и $\lambda_{k i}(\omega)$ задаются равенствами (18) и (38) соответственно). Используя неравенство Коши-Буняковского, из условия $F_{3}$ находим, что существует константа $K_{1}>0$ такая, что $\lambda_{k i}(\omega) \leqslant K_{1}$ для всех $k=1, \ldots, r, i=1, \ldots, N$ и $\omega=1,2, \ldots$ Следовательно, из любой бесконечной последовательности $\left\{\omega^{\prime}\right\}$ значений параметра $\omega$, извлеченной без возвращения из исходной последовательности $\omega=1,2, \ldots$, можно выбрать (без возвращения) бесконечную подпоследовательность $\left\{\omega^{\prime \prime}\right\}$, для которой существуют пределы

$$
\lim _{\omega^{\prime \prime} \rightarrow \infty} \lambda_{k i}\left(\omega^{\prime \prime}\right)=\lambda_{k i}<\infty, \quad k=1, \ldots, r, \quad i=1, \ldots, N .
$$

При этом из (37) и (41) получаем равенства

$$
\sum_{i=1}^{N} \lambda_{k i} \lambda_{l i}=\alpha_{k l}, \quad k, l=1, \ldots, r .
$$

Заметим, что из (42) следует, что в условиях теоремы 2 все величины $\alpha_{k l}$ конечны.

Для каждого $\omega^{\prime \prime} \in\left\{\omega^{\prime \prime}\right\}$ выполняется альтернатива $H\left(\bar{p}_{\omega^{\prime \prime}}\right)$ к гипотезе $H_{0}$. Принимая во внимание лемму 3 , равенство (40) и существование пределов (41), приходим к выводу, что случайный вектор $\tilde{\xi}=\tilde{\xi}\left(\omega^{\prime \prime}\right)$ с компонентами (2) при $\omega^{\prime \prime} \rightarrow \infty$ сходится по распределению к нормальному случайному вектору

$$
\xi_{2}=\left(\xi_{11}^{(2)}, \ldots, \xi_{1 N}^{(2)} ; \ldots ; \xi_{r 1}^{(2)}, \ldots, \xi_{r N}^{(2)}\right)
$$

для компонент которого выполняются равенства

$$
\mathbf{E} \xi_{k i}^{(2)}=\lambda_{k i}, \quad k=1, \ldots, r, \quad i=1, \ldots, N,
$$

где $\lambda_{k i}$ определяются соотношениями (41). Ковариационной матрицей случайного вектора (43) является матрица $D$ из леммы 2. Согласно векторной версии предложения XII из [8] (см. [8], п. 2 , с. 4 ) в условиях теоремы 2 при $\omega^{\prime \prime} \rightarrow \infty$ статистика $\chi^{2}\left(\omega^{\prime \prime}\right)$ имеет предельное распределение, преобразование Лапласа которого равно

$$
\begin{aligned}
L_{r}\left(t_{1}, \ldots, t_{r}\right) & =\lim _{\omega^{\prime \prime} \rightarrow \infty} \mathbf{E} \exp \left\{-\sum_{k=1}^{r} t_{k} \chi_{k}^{2}\left(\omega^{\prime \prime}\right)\right\} \\
& =\operatorname{E} \exp \left\{-\sum_{k=1}^{r} t_{k} \sum_{i=1}^{N}\left(\xi_{k i}^{(2)}\right)^{2}\right\},
\end{aligned}
$$


где случайные величины $\xi_{k i}^{(2)}-$ компоненты случайного вектора (43).

Воспользуемся матрицей $C$, приведенной в доказательстве теоремы 1 , и для $i=1, \ldots, N$ положим

$$
\eta_{i k}^{(1)}=\sum_{i=1}^{N} c_{i j} \xi_{k j}^{(2)}, \quad k=1, \ldots, r, \quad \eta_{i}^{(1)}=\left(\eta_{i 1}^{(1)}, \ldots, \eta_{i r}^{(1)}\right) .
$$

Из (45) и ортогональности матрицы $C$ следует, что

$$
L_{r}\left(t_{1}, \ldots, t_{r}\right)=\mathbf{E} \exp \left\{-\sum_{k=1}^{r} t_{k} \sum_{i=1}^{N}\left(\eta_{i k}^{(1)}\right)^{2}\right\}
$$

Как и при доказательстве теоремы 1 , учитывая вид матрицы $D$, которая является ковариационной матрицей нормального случайного вектора (43), убеждаемся, что случайный вектор $\eta_{N}^{(1)}$ с вероятностью 1 равен нулю, а векторы $\eta_{1}^{(1)}, \ldots, \eta_{N-1}^{(1)}$ независимы, и каждый из них распределен по $r$-мерному нормальному закону с ковариационной матрицей $R$. Далее, из (44) и (46) находим, что

$$
m_{i k}=\mathbf{E} \eta_{i k}^{(1)}=\sum_{j=1}^{N} c_{i j \lambda_{k j}}, \quad k=1, \ldots, r, \quad i=1, \ldots, N,
$$

так что $\eta_{i}^{(1)}$ имеет вектор средних значений $m_{i}=\left(m_{i 1}, \ldots, m_{i r}\right), i=1, \ldots, N$, причем $m_{N}=0$. Применяя лемму 4, из (47) получаем, что

$$
L_{r}\left(t_{1}, \ldots, t_{r}\right)=|I+2 T R|^{-(N-1) / 2} \exp \left\{-\sum_{k, l=1}^{r} \sum_{i=1}^{N-1} m_{i k} m_{i l} q_{k l}\right\},
$$

где $q_{k l}, k, l=1, \ldots, r$ - элементы матрицы (31). Согласно (46) для $k=1, \ldots, r$ вектор $\left(\lambda_{k 1}, \ldots, \lambda_{k N}\right)$ переводится в вектор $\left(m_{1 k}, \ldots, m_{N k}\right)$ ортогональным преобразованием. Поэтому ввиду (42) имеют место равенства

$$
\sum_{i=1}^{N-1} m_{i k} m_{i l}=\sum_{i=1}^{N} \lambda_{k i} \lambda_{l i}=\alpha_{k l}, \quad k, l=1, \ldots, r .
$$

В итоге выражение (48) для $L_{r}\left(t_{1}, \ldots, t_{r}\right)$ приводится к виду (39), и оно не зависит от подпоследовательности $\omega^{\prime \prime}$. Вследствие этого предельное при $\omega^{\prime \prime} \rightarrow \infty$ распределение статистики $\chi^{2}\left(\omega^{\prime \prime}\right)$ одно и то же для всех возможных подпоследовательностей $\left\{\omega^{\prime \prime}\right\}$, выбираемых указанным выше способом. Следовательно, можно воспользоваться теоремой о сходимости вероятностных мер (см. [9], с. 27) и с ее помощью завершить доказательство теоремы 2.

Из доказательства теоремы 2 следует совместность уравнений (30), в которых величины $\alpha_{k l}$ задаются соотношениями (37). Поэтому из (39) и леммы 4 вытекает, что в условиях теоремы 7, если в (37) не все величины $\alpha_{k l}$ равны нулю, предельное распределение статистики $\chi^{2}$ есть многомерное нецентральное распределение хи-квадрат с параметрами $r$, $N-1, R=\left\|\rho_{k l}\right\|_{k, l=1}^{r}$ и $\alpha_{k l}, 1 \leqslant k \leqslant l \leqslant r$. 
В некоторых случаях утверждение теоремы 2 можно упростить. Предположим, что для полиномиальных схем (6), определяющих $H(\bar{p})$, для $m \in A$ выполняется равенство $\bar{p}_{m}=q$, где $q=q_{\omega}=\left(q_{1}, \ldots, q_{N}\right), 0 \leqslant q_{i} \leqslant 1, i=1, \ldots, N, \sum_{i=1}^{N} q_{i}=1$. Соответствующую простую гипотезу относительно распределений случайных величин (1) обозначим $H(q)=H(q(\omega))$.

Следствие 2. Пусть случайные величины (1) получены в предположении, что верна альтернатива $H\left(q_{\omega}\right)$ к гипотезе $H_{0}$, выполнены условия $F_{1}, F_{2}$ и существуют пределы

$$
\begin{gathered}
\lim _{\omega \rightarrow \infty} \frac{\Delta_{k}}{\Delta}=\beta_{k}^{2}, \quad k=1, \ldots, r, \\
\lim _{\omega \rightarrow \infty} \Delta \sum_{i=1}^{N} \frac{1}{p_{i}}\left(q_{i}-p_{i}\right)^{2}=\gamma^{2}<\infty .
\end{gathered}
$$

Тогда при $\omega \rightarrow \infty$ статистика (4) имеет предельное распределение, преобразование Лапласа которого имеет вид

$$
L_{r}\left(t_{1}, \ldots, t_{r}\right)=|I+2 T R|^{(N-1) / 2} \exp \left\{-\gamma^{2} b Q b^{\prime}\right\},
$$

где $t_{k} \geqslant 0, k=1, \ldots, r, b=\left(\beta_{1}, \ldots, \beta_{r}\right) u Q-$ матрича $(31)$, причем $R=\left\|\rho_{k l}\right\|_{k, l=1}^{r}$.

Доказательство. В случае справедливости альтернативы $H\left(q_{\omega}\right)$ для величин (33) имеют место равенства

$$
\lambda_{k i}(\omega)=\sqrt{\Delta_{k} / p_{i}}\left(q_{i}-p_{i}\right), \quad k=1, \ldots, r, \quad i=1, \ldots, N .
$$

Отсюда, учитывая (49) и (50), находим, что

$$
\lim _{\omega \rightarrow \infty} \sum_{i=1}^{N} \lambda_{k i}(\omega) \lambda_{l i}(\omega)=\gamma_{2} \beta_{k} \beta_{l}, \quad k, l=1, \ldots, r .
$$

В силу (50) выполняется условие $F_{3}$. Поэтому к статистике (4) можно применить теорему 2 , вследствие которой при $\omega \rightarrow \infty$ статистика $\chi^{2}$ имеет предельное распределение и его преобразование Лапласа в силу (37), (39) и (52) имеет вид (51). Следствие доказано.

Следствие 3. Пусть выполнены предположения теоремы 2, но вместо пределов (37) существуют пределы (49), $и$

$$
\lim _{\omega \rightarrow \infty} \sqrt{\Delta / \Delta_{k}} \lambda_{k i}(\omega)=\gamma_{k i}, \quad k=1, \ldots, r, \quad i=1, \ldots, N,
$$

где величины $\lambda_{k i}(\omega)$ определены равенствами (38). Тогда при $\omega \rightarrow \infty$ статистика (4) имеет предельное распределение, преобразование Лапласа которого имеет вид

$$
\begin{aligned}
L_{r}\left(t_{1}, \ldots, t_{r}\right) & =|I+2 T R|^{-(N-1) / 2} \exp \left\{-\sum_{i=1}^{N} g_{i} Q g_{i}^{\prime}\right\} \\
& =|I+2 T R|^{-(N-1) / 2} \exp \left\{-\sum_{k=l=1}^{r}\left(\sum_{i=1}^{N} \gamma_{k i} \gamma_{l i}\right) \beta_{k} \beta_{l} q_{k l}\right\},
\end{aligned}
$$

где $t_{k} \geqslant 0, k=1, \ldots, r, g_{i}=\left(\beta_{1} \gamma_{1 i}, \ldots, \beta_{r} \gamma_{r i}\right), i=1, \ldots, N, u Q=\left\|q_{k l}\right\|_{k, l=1}^{r}$ матрича (31), причем $R=\left\|\rho_{k l}\right\|_{k, l=1}^{r}$.

Частными случаями теоремы 1 , доказанной выше, является теорема 1 из [1] и утверждения теорем 1 и 2 из [2], а из следствия 3 для статистики последовательного критерия хи-квадрат можно вывести теорему 2 из [1] (случай близких альтернатив). 


\section{Список литературы}

1. Захаров В. К., Сарманов О. В., Севастьянов Б. А., Последовательный критерий $\chi^{2}$. Матем. сб. (1969) 79, №3, 444-460.

2. Тихомирова М. И., Чистяков В. П., Скользящий хи-квадрат. Дискретная математика (2000) 12 , №4, 46-52.

3. Jensen D. R., Limit properties on noncentral multivariate Rayleigh and chi-square distributions. SIAM J. Appl. Math. (1969) 17, №4, 802-814.

4. Гаек Я., Шидак 3., Теория ранговых критериев. Наука, Москва, 1971.

5. Селиванов Б. И., Об одном классе статистик типа хи-квадрат. Обозрение прикладной и промышленной математики (1995) 2, №6, 926-966.

6. Гнеденко Б. В., Элементы теории функций распределения случайных векторов. Успехи матем. наук (1944) 10, 230-244.

7. Барра Ж.-Р., Основные понятия математической статистики. Мир, Москва, 1974.

8. Рао С. Р., Линейные статистические методы. Наука, Москва, 1968.

9. Биллингсли П., Сходимость вероятностных мер. Наука, Москва, 1974.

Статья поступила 30.01.2002. 